\title{
Serum concentrations of retinol-binding protein 4 in women with and without gestational diabetes
}

\author{
K. Krzyzanowska $\cdot$ L. Zemany $\cdot$ W. Krugluger • \\ G. H. Schernthaner $\cdot$ F. Mittermayer $\cdot$ C. Schnack • \\ R. Rahman • J. Brix • B. B. Kahn • G. Schernthaner
}

Received: 21 December 2007 / Accepted: 18 March 2008 / Published online: 25 April 2008

(C) Springer-Verlag 2008

\begin{abstract}
Aims/hypothesis Pregnancy is characterised by temporarily increased insulin resistance. Gestational diabetes occurs when pancreatic beta cell function is unable to compensate for this insulin resistance. Retinol-binding protein 4 (RBP4) could be related to insulin resistance. We hypothesised that RBP4 is elevated in gestational diabetes.

Methods Serum RBP4, transthyretin and retinol were crosssectionally measured in 42 women with gestational diabetes and 45 pregnant controls. Of these, 20 women with and 22 without gestational diabetes were included in an additional
\end{abstract}

K. Krzyzanowska $(\bowtie) \cdot$ C. Schnack $\cdot$ R. Rahman

J. Brix $\cdot$ G. Schernthaner

Department of Internal Medicine I,

Rudolfstiftung Hospital,

Juchgasse 25,

1030 Vienna, Austria

e-mail: katarzyna.krzyzanowska@wienkav.at

L. Zemany • B. B. Kahn

Division of Endocrinology,

Diabetes and Metabolism,

Beth Israel Deaconess Medical Center,

Harvard Medical School,

Boston, MA, USA

W. Krugluger

Central Laboratory, Rudolfstiftung Hospital,

Vienna, Austria

G. H. Schernthaner

Department of Internal Medicine II,

Medical University Vienna,

Vienna, Austria

F. Mittermayer

Department of Clinical Pharmacology,

Medical University Vienna,

Vienna, Austria longitudinal study. RBP4 was determined by enzyme immunometric assay (EIA) and western blot.

Results Women with gestational diabetes had lower RBP4 EIA and western blot levels than controls (median 6.8 [interquartile range, 3.9-14.3] vs 11.3 [7.8-19.9] $\mu \mathrm{g} / \mathrm{ml}, p<0.001$ and 25.1 [21.7-29.6] vs $26.6[23.5-32.2] \mu \mathrm{g} / \mathrm{ml}, p=0.026)$. Transthyretin and the RBP4:transthyretin molar ratio were comparable between the groups. Serum retinol was lower $(p<0.001)$ and the RBP4 Western blot level: retinol molar ratio was higher in women with gestational diabetes $(p=0.044)$. RBP4 was not associated with the glucose or homeostasis model assessment of insulin resistance (HOMA-IR), but in gestational diabetes the RBP4:retinol molar ratio correlated with blood glucose and negatively with $2 \mathrm{~h}$ post-load insulin. The RBP4:transthyretin ratio correlated with HOMA-IR and fasting insulin in controls. In women with gestational diabetes RBP4 EIA and western blot levels increased after delivery. Retinol increased in both groups, while transthyretin and the RBP4:transthyretin ratio were not altered after parturition.

Conclusions/interpretation RBP4 measured by two different techniques is not elevated, but the RBP4:retinol molar ratio is higher and correlates with fasting blood glucose in women with gestational diabetes. Thus, the RBP4:retinol ratio and the RBP4:transthyretin ratio are more informative than RBP4 levels alone when assessing insulin-glucose homeostasis during pregnancy.

Keywords Gestational diabetes mellitus - Insulin resistance · Retinol-binding protein $4 \cdot$ Transthyretin

$\begin{array}{ll}\text { Abbreviations } \\ \text { EIA } & \begin{array}{l}\text { enzyme immunometric assay } \\ \text { homeostasis model assessment of insulin } \\ \text { HOMA-IR }\end{array} \\ \text { RBP4 } & \begin{array}{l}\text { retinol-binding protein } 4 \\ \text { retinol }\end{array}\end{array}$




\section{Introduction}

Pregnancy is associated with progressive insulin resistance, which begins during mid-pregnancy and progresses throughout the third trimester. Gestational diabetes mellitus is defined as glucose intolerance with onset or first recognition during pregnancy [1]. Women with gestational diabetes show various features of the metabolic syndrome [2] like obesity, low-grade inflammation [3] or hypertriacylglycerolaemia [4, 5], and have an increased risk of developing type 2 diabetes mellitus [6]. Insulin resistance plays a major role in the adverse cardiovascular risk profile found in women with gestational diabetes. However, insulin resistance during pregnancy is not fully explained by obesity [7]. Multiple factors like alterations of the insulin-signalling pathway [8], increased circulating leptin [9], reduced adiponectin [10] and inflammation [11, 12] could lead to exaggerated insulin resistance during gestational diabetes pregnancies $[8,13]$. In addition, during pregnancy, insulin secretion from pancreatic beta cells increases to compensate for the insulin resistance. During the third trimester, the greatest metabolic difference between control women and women with gestational diabetes may be insulin levels or insulin secretion [14].

Humans with insulin resistance show a downregulation of the insulin-stimulated GLUT4, which leads to impaired insulin-stimulated glucose uptake in adipocytes $[15,16]$. In adipocytes of women with gestational diabetes, GLUT4 levels may be reduced or the insulin-stimulated recruitment of GLUT4 to the plasma membrane may be impaired [17]. As shown by Yang et al. [18], genetic knockout of Glut4 (also known as Slc2a4) results in increased production of the adipokine, retinol-binding protein 4 (RBP4), in adipose tissue. Experimental elevation of RBP4 was demonstrated to impair insulin-signalling and induce gluconeogenic enzymes in the liver [18]. In line with these experimental findings, patients with obesity and type 2 diabetes were found to have elevated RBP4 serum concentrations [19, 20]. Thus, RBP4 might contribute to the development of insulin resistance in vivo.

To evaluate whether the temporary state of increased insulin resistance in women with gestational diabetes is related to serum RBP4 concentrations, we determined RBP4 in women with gestational diabetes and in pregnant healthy women. To gather information on the course of RBP4 concentrations after normalisation of the glucose tolerance state, we measured this adipokine during pregnancy and after delivery in a subgroup of women with gestational diabetes and healthy pregnant controls. RBP4 circulates in a complex with transthyretin, which maintains serum RBP4 levels by preventing renal clearance of RBP4 [21, 22]. Since alterations in transthyretin levels could affect RBP4 levels, transthyretin was measured at all time points in both groups of women. Moreover, since RBP4 is a transport protein for retinol and must be bound to retinol to be secreted at least from liver [23], retinol levels were measured in all participants.

\section{Methods}

Participants and study protocol All clinical investigations were conducted in accordance with the guidelines in The Declaration of Helsinki and approved by the institutional review committee. All participants were carefully instructed about the aims of the study and written informed consent was given.

All participants were non-smokers. Gestational diabetes was diagnosed according to the criteria of the 4th Workshop Conference of Gestational Diabetes [24]. Insulin resistance was estimated by homeostasis model assessment of insulin resistance (HOMA-IR) [25]. In women with gestational diabetes who were on insulin therapy, HOMA-IR was assessed before initiation of insulin therapy.

Cross-sectional protocol A total of 42 women with gestational diabetes and 45 pregnant healthy controls were included in this study. Of those with gestational diabetes, 11 women were treated with insulin and 31 with diet alone. After an overnight fast, serum samples were obtained at median week 29 in gestational diabetes patients and at median gestational week 29 in controls.

Longitudinal protocol From the cross-sectional cohort, 20 women with gestational diabetes and 22 pregnant control women were also included into the longitudinal study. Serum samples were obtained at median gestational week 30 in gestational diabetes patients and at median gestational week 30 in controls. Additional samples were taken in both groups at 8 weeks after delivery, following an overnight fast.

Laboratory analysis Glucose, insulin, triacylglycerol and $\mathrm{HbA}_{1 \mathrm{c}}$ were determined with standard laboratory methods. Retinol concentrations were measured using HPLC. Glomerular filtration rate was estimated using the Modification of Diet in Renal Disease (MDRD) formula [26]. To overcome known shortcomings in RBP4 measurements, we used two different methods to determine RBP4 levels [27]. The first method was an enzyme immunometric assay (EIA) (RBP4 EIA kit; Phoenix Pharmaceuticals, Belmont, CA, USA), in which the inter- and intra-assay variability for RBP 4 are $\leq 14$ and $\leq 5 \%$, respectively. The second method for RBP4 quantification was quantitative western blotting using protein standards prepared with purified full-length human recombinant RBP4. Transthyretin was measured on the same gels by a similar quantitative western blotting method, using transthyretin protein standards purified from human plasma (Sigma, St Louis, MO, USA). The coefficient of variation for 
interassay replicate samples was less than $10 \%$ by this method [18].

Statistical analyses Skewed variables (RBP4, transthyretin, retinol, insulin, HOMA-IR, triacylglycerol, GFR and $\mathrm{HbA}_{1 \mathrm{c}}$ ) were log-transformed for all statistical analyses. Differences between participants with and without gestational diabetes were analysed with unpaired Student's $t$ tests and correlations calculated using Pearson correlations. Correction for potential confounders was performed with analysis of covariance. The longitudinal study included only women with examinations during and after pregnancy $(n=20$ for gestational diabetes; $n=22$ for control). ANOVA and the Fisher least significant difference post hoc test were applied to test for differences between gestational diabetes patients and controls, and for changes in RBP4 and transthyretin after delivery in the longitudinal protocol. Statistica software version 6.0 (StatSoft, Tulsa, OK, USA) was used for all analyses. A value of $p<0.05$ was considered the level of significance. Data are presented as medians (interquartile range). According to a power calculation assuming an alpha of 0.05 and sigma of 5 , a study with 59 participants has $90 \%$ power to detect a $3 \mu \mathrm{g} / \mathrm{ml}$ difference in RBP4 concentrations. Therefore we aimed to include at least 60 participants in this study.

\section{Results}

Cross-sectional protocol During gestation, women with gestational diabetes had significantly lower RBP4 levels than healthy pregnant controls as measured by EIA and marginally lower RBP4 as measured by western blot. Although both methods showed a similar trend, there was a considerable discrepancy in the magnitude of the effect between the two assays, possibly reflecting different affinities of the antibodies for different forms of RBP4 [27]. Transthyretin concentrations and the RBP4:transthyretin molar ratio did not differ between the study groups. Importantly, women with gestational diabetes had lower retinol concentrations and higher western blot RBP4:retinol molar ratio than pregnant controls (Table 1).

Patients with gestational diabetes were older and had higher HOMA-IR, fasting and post-load glucose, $\mathrm{HbA}_{1 \mathrm{c}}$ and BMI before and during pregnancy. Fasting insulin, diastolic blood pressure, triacylglycerol and GFR were comparable between both groups (Table 1). After adjusting for age and BMI, the HOMA-IR was not different between participants with gestational diabetes and controls $(p=0.116)$. After controlling for age and BMI during pregnancy, both RBP4 EIA and RBP4 western blot levels remained significantly lower in participants with gestational diabetes $(p<0.001$ and $p=0.016)$. After additional adjustment for retinol
Table 1 RBP4 and clinical parameters in patients with and without gestational diabetes mellitus

\begin{tabular}{|c|c|c|c|}
\hline & $\begin{array}{l}\text { Gestational } \\
\text { diabetes }(n=42)\end{array}$ & $\begin{array}{l}\text { No gestational } \\
\text { diabetes }(n=45)\end{array}$ & $\begin{array}{l}p \\
\text { value }\end{array}$ \\
\hline $\begin{array}{l}\text { EIA RBP4 } \\
(\mu \mathrm{g} / \mathrm{ml})\end{array}$ & $6.8(3.9-14.3)$ & $11.3(7.8-19.9)$ & $<0.001$ \\
\hline $\begin{array}{l}\text { Western blot } \\
\text { RBP4 }(\mu \mathrm{g} / \mathrm{ml})\end{array}$ & $25.1(21.7-29.6)^{\mathrm{a}}$ & $26.6(23.5-32.2)$ & 0.026 \\
\hline $\begin{array}{l}\text { Transthyretin } \\
(\mu \mathrm{g} / \mathrm{ml})\end{array}$ & $144.5(126.2-191.3)^{\mathrm{a}}$ & $146.4(127.5-200.6)$ & 0.228 \\
\hline $\begin{array}{l}\text { RBP4: } \\
\text { transthyretin }^{\text {b }}\end{array}$ & $0.40(0.35-0.54)^{\mathrm{a}}$ & $0.41(0.36-0.57)$ & 0.522 \\
\hline $\begin{array}{l}\text { Retinol } \\
(\mu \mathrm{mol} / \mathrm{l})\end{array}$ & $0.97(0.76-1.10)^{\mathrm{a}}$ & $1.26(0.88-1.64)$ & $<0.001$ \\
\hline RBP4:retinol ${ }^{\mathrm{c}}$ & $1.29(1.03-1.62)$ & $1.13(0.73-1.50)$ & 0.044 \\
\hline Age (years) & $33(29-35)$ & $28(24-34)$ & 0.020 \\
\hline $\begin{array}{l}\text { BMI before } \\
\text { pregnancy } \\
\left(\mathrm{kg} / \mathrm{m}^{2}\right)\end{array}$ & $29(25-34)$ & $23(22-27)$ & $<0.001$ \\
\hline $\begin{array}{l}\text { BMI during } \\
\text { pregnancy } \\
\left(\mathrm{kg} / \mathrm{m}^{2}\right)\end{array}$ & $34(29-38)$ & $29(25-31)$ & $<0.001$ \\
\hline $\begin{array}{l}\text { Systolic BP } \\
(\mathrm{mmHg})\end{array}$ & $117(111-127)$ & $112(104-120)$ & 0.060 \\
\hline $\begin{array}{l}\text { Diastolic BP } \\
(\mathrm{mmHg})\end{array}$ & $73(67-78)$ & $73(66-78)$ & 0.889 \\
\hline $\begin{array}{l}\text { Fasting insulin } \\
(\mathrm{pmol} / \mathrm{l})\end{array}$ & $98(73-137)$ & $89(53-124)$ & 0.086 \\
\hline $\begin{array}{l}1 \mathrm{~h} \text { Insulin } \\
(\mathrm{pmol} / \mathrm{l})\end{array}$ & $597(430-848)$ & $715(354-1168)$ & 0.746 \\
\hline $\begin{array}{l}2 \text { h Insulin } \\
(\mathrm{pmol} / \mathrm{l})\end{array}$ & $810(90.9-631)$ & $602(354-1000)$ & 0.069 \\
\hline HOMA-IR & $3.4(2.2-4.5)$ & $2.0(1.4-3.4)$ & $<0.001$ \\
\hline $\begin{array}{l}\text { Fasting glucose } \\
(\mathrm{mmol} / \mathrm{l})\end{array}$ & $5.4(4.7-5.8)$ & $4.1(3.7-4.3)$ & $<0.001$ \\
\hline $\begin{array}{l}1 \mathrm{~h} \text { post-load } \\
\text { glucose } \\
(\mathrm{mmol} / \mathrm{l})\end{array}$ & $12.0(11.0-13.3)$ & $7.7(6.6-8.9)$ & $<0.001$ \\
\hline $\begin{array}{l}2 \mathrm{~h} \text { post-load } \\
\text { glucose } \\
(\mathrm{mmol} / \mathrm{l})\end{array}$ & $9.7(8.9-10.6)$ & $6.5(5.6-7.2)$ & $<0.001$ \\
\hline $\begin{array}{l}\text { Triacylglycerol } \\
(\mathrm{mmol} / \mathrm{l})\end{array}$ & $2.2(1.8-1.7)$ & $2.0(1.5-1.2)$ & 0.289 \\
\hline $\begin{array}{l}\text { GFR }(\mathrm{ml} \mathrm{min}-1 \\
\left.1.73 \mathrm{~m}^{-2}\right)\end{array}$ & $127(121-150)$ & $145(120-161)$ & 0.128 \\
\hline $\mathrm{HbA}_{1 \mathrm{c}}(\%)$ & $5.5(5.3-5.8)$ & $5.2(4.9-5.6)$ & $<0.001$ \\
\hline
\end{tabular}

Data are presented as medians (interquartile range); all data were collected after an overnight fast at week 29 (median) in both participant groups.

Student's $t$ tests were applied to test for significant differences between groups after log-transformation of skewed variables

${ }^{\text {a }}$ Only performed in 41 patients

${ }^{\mathrm{b}}$ Molar ratio, according to the amounts of RBP4 and transthyretin determined by western blotting, calculated using a molecular mass of $21 \mathrm{kDa}$ for RBP4 and $54 \mathrm{kDa}$ for transthyretin as a homotetramer $(n=19)$

${ }^{\mathrm{c}}$ Molar ratio, according to the amount of RBP4 determined by western blotting and the amount of retinol determined by HPLC, calculated using a molecular mass of $21 \mathrm{kDa}$ for RBP4 and $0.286 \mathrm{kDa}$ for retinol $(n=19)$ 
concentrations, the observed difference in RBP4 EIA levels remained, while the difference in western blot RBP4 levels was lost $(p=0.008$ and $p=0.153)$.

In women with gestational diabetes, the RBP4:retinol molar ratio as determined by EIA and western blot correlated with fasting blood glucose (RBP4 EIA $R=0.32$, $p=0.04$; RBP4 western blot $\mathrm{R}=0.34, p=0.031)$. The western blot RBP4:retinol molar ratio correlated negatively with $2 \mathrm{~h}$ post-load insulin levels. RBP4 EIA levels correlated with systolic blood pressure. Western blot RBP4 levels correlated with retinol and systolic blood pressure, but not with other clinical parameters in women with gestational diabetes. In healthy controls, RBP4 EIA levels correlated with retinol and systolic blood pressure, while western blot RBP4 levels were associated with diastolic blood pressure and triacylglycerol. The RBP4:transthyretin molar ratio correlated significantly with systolic blood pressure in women with gestational diabetes. In healthy controls, BMI during pregnancy, fasting insulin and HOMA-IR were significantly related to the RBP4:transthyretin molar ratio (Table 2). To exclude the possibility of confounding effects by insulin treatment, we performed an analysis of RBP4 and insulin therapy: EIA and western blot RBP4 levels did not differ between women with and without insulin therapy (6.7 [interquartile range 3.9-14.1] vs $10.6[5.8-17.9] \mu \mathrm{g} / \mathrm{ml}, p=0.123$ and $24.0[19.5-30.7]$ vs $26.5[22.9-30.4] \mu \mathrm{g} / \mathrm{ml}, p=0.319)$.

Longitudinal protocol Regarding clinical characteristics and outcome parameters, no significant differences could be observed between women included and those not included in the longitudinal protocol (data not shown). In women with gestational diabetes, RBP4 EIA levels increased after delivery $(p=0.005)$ and a minimal increase of western blot RBP4 levels was seen $(p=0.013)$. In the healthy pregnant controls, no significant change in EIA and western blot RBP4 levels was observed $(p=0.637$ and $p=0.528)$. During pregnancy, RBP4 EIA levels were lower in women with gestational diabetes than in controls $(p=0.026)$, while western blot RBP4 levels did not differ significantly between the groups $(p=0.206)$. After delivery, EIA and western blot RBP4 levels were comparable between women with gestational diabetes and controls ( $p=0.715$ and $p=0.473$ ). No between-group differences in transthyretin or the RBP4:transthyretin molar ratio before and after delivery were found; similarly, no significant longitudinal changes in transthyretin or the RBP4:transthyretin

Table 2 Pearson correlation coefficients for various RBP4 measurements and other parameters as indicated in patients with and without gestational diabetes mellitus

\begin{tabular}{|c|c|c|c|c|c|c|c|c|}
\hline & \multicolumn{2}{|l|}{ RBP4 EIA } & \multicolumn{2}{|c|}{ RBP4 western blot } & \multicolumn{2}{|c|}{ RBP4:transthyretin ${ }^{a}$} & \multicolumn{2}{|l|}{ RBP4:retinol ${ }^{\mathrm{b}}$} \\
\hline & $\operatorname{GDM}(n=42)$ & $\begin{array}{l}\text { No GDM } \\
(n=45)\end{array}$ & $\operatorname{GDM}(n=41)$ & $\begin{array}{l}\text { No GDM } \\
(n=45)\end{array}$ & $\operatorname{GDM}(n=41)$ & $\begin{array}{l}\text { No GDM } \\
(n=45)\end{array}$ & $\operatorname{GDM}(n=41)$ & $\begin{array}{l}\text { No GDM } \\
(n=45)\end{array}$ \\
\hline Retinol & $0.09(0.575)$ & $0.33(0.034)$ & $0.48(0.002)$ & $0.11(0.495)$ & $0.10(0.534)$ & $0.10(0.516)$ & $-0.65(<0.001)$ & $-0.87(<0.001)$ \\
\hline Age & $0.01(0.959)$ & $0.24(0.112)$ & $-0.22(0.170)$ & $0.15(0.341)$ & $0.02(0.879)$ & $-0.05(0.720)$ & $-0.19(0.244)$ & $0.01(0.983)$ \\
\hline $\begin{array}{l}\text { BMI pre- } \\
\text { pregnancy }^{\mathrm{c}}\end{array}$ & $0.13(0.404)$ & $-0.11(0.477)$ & $0.10(0.557)$ & $0.05(0.732)$ & $0.28(0.082)$ & $0.28(0.067)$ & $0.13(0.422)$ & $-0.21(0.189)$ \\
\hline $\mathrm{BMI}_{\text {pregnancy }}{ }^{\mathrm{c}}$ & $0.14(0.362)$ & $-0.03(0.847)$ & $0.19(0.232)$ & $0.10(0.517)$ & $0.27(0.088)$ & $0.36(0.017)$ & $0.06(0.422)$ & $-0.25(0.110)$ \\
\hline Systolic BP & $0.33(0.042)$ & $0.32(0.034)$ & $0.34(0.039)$ & $0.21(0.178)$ & $0.41(0.010)$ & $0.18(0.249)$ & $0.08(0.626)$ & $0.704)$ \\
\hline Diastolic BP & $0.25(0.116)$ & & & & $0.21(0.199)$ & $0.19(0.210)$ & $0.05(0.782)$ & $0.801)$ \\
\hline Fasting insulin & $0.15(0.358)$ & $0.11(0.475)$ & $0.14(0.39)$ & $0.28(0.069)$ & $0.14(0.387)$ & $0.42(0.004)$ & $-0.01(0.989)$ & $-0.02(0.891)$ \\
\hline $1 \mathrm{~h}$ insulin & $0.18(0.335)$ & $0.05(0.756)$ & $0.30(0.096)$ & $-0.07(0.649)$ & $0.25(0.165)$ & $-0.02(0.895)$ & $-0.09(0.641)$ & $(0.314)$ \\
\hline $2 \mathrm{~h}$ insulin & $-0.01(0.998)$ & $0.14(0.377)$ & $-0.01(0.928)$ & $0.21(0.193)$ & $-0.02(0.928)$ & $0.31(0.047)$ & $-0.39(0.028)$ & $0.03(0.853)$ \\
\hline HOMA-IR & $0.19(0.230)$ & $0.10(0.518)$ & $0.15(0.354)$ & $0.28(0.061)$ & $0.22(0.169)$ & $0.39(0.008)$ & $0.07(0.671)$ & $0.01(0.975)$ \\
\hline Fasting glucose & $0.27(0.083)$ & $0.03(0.863)$ & $0.13(0.409)$ & $0.16(0.287)$ & $0.14(0.387)$ & $0.01(0.950)$ & $0.34(0.031)$ & $0.08(0.636)$ \\
\hline $1 \mathrm{~h}$ glucose & $0.08(0.610)$ & $-0.04(0.807)$ & $0.08(0.636)$ & $-0.03(0.826)$ & $-0.13(0.414)$ & $-0.12(0.439)$ & $0.28(0.075)$ & $-0.15(0.339)$ \\
\hline $2 \mathrm{~h}$ glucose & $0.16(0.310)$ & $-0.01(0.945)$ & $-0.19(0.243)$ & $0.28(0.070)$ & $-0.30(0.059)$ & $0.21(0.164)$ & $-0.07(0.671)$ & $0.06(0.693)$ \\
\hline Triacylglycerol & $0.25(0.114)$ & $0.06(0.690)$ & $-0.03(0.840)$ & $0.35(0.024)$ & $-0.05(0.758)$ & $0.23(0.149)$ & $-0.10(0.534)$ & $0.08(0.620)$ \\
\hline GFR & $0.02(0.920)$ & $-0.22(0.154)$ & $0.03(0.839)$ & $-0.07(0.640)$ & $-0.09(0.594)$ & $-0.07(0.662)$ & $0.26(0.103)$ & $0.16(0.337)$ \\
\hline $\mathrm{HbA}_{1 \mathrm{c}}$ & $0.24(0.125)$ & $0.05(0.763)$ & $-0.10(0.545)$ & $0.23(0.137)$ & $0.23(0.149)$ & $0.26(0.091)$ & $0.25(0.121)$ & $0.25(0.125)$ \\
\hline
\end{tabular}

Values are Pearson correlation coefficients ( $p$ value)

All data were collected after an overnight fast at median week 29 in both participant groups; skewed variables were log-transformed

${ }^{a}$ Molar ratio, according to the amounts of RBP4 and transthyretin determined by western blotting, calculated using a molecular mass of $21 \mathrm{kDa}$ for RBP4 and $54 \mathrm{kDa}$ for transthyretin as a homotetramer $(n=19)$

${ }^{\mathrm{b}}$ Molar ratio, according to the amount of RBP4 determined by western blotting and the amount of retinol determined by HPLC, calculated using a molecular mass of $21 \mathrm{kDa}$ for RBP4 and $0.286 \mathrm{kDa}$ for retinol $(n=19)$

${ }^{c}$ Pre-pregnancy BMI was compared with RBP4 values obtained at median week 29 of pregnancy 
molar ratio could be found. After parturition, retinol concentrations increased in both groups, but were higher in women with gestational diabetes. The RBP4:retinol molar ratio decreased in women with and without gestational diabetes after delivery, and was lower in the former.

BMI was significantly higher in women with gestational diabetes and decreased in both groups after delivery. After parturition, 1 and $2 \mathrm{~h}$ post-load glucose decreased significantly in women with gestational diabetes. Fasting glucose increased and $1 \mathrm{~h}$ post-load glucose decreased after delivery in healthy pregnant controls. During pregnancy, fasting and 1 and $2 \mathrm{~h}$ post-load glucose values were higher in women with gestational diabetes than in controls. After delivery women with gestational diabetes had elevated fasting and $1 \mathrm{~h}$ post-load glucose compared with controls. Triacylglycerol and GFR decreased in all women after delivery (Table 3).

\section{Discussion}

This is one of the first studies describing the novel adipocyteand liver-secreted protein RBP4 in women with gestational diabetes and pregnant healthy controls. Total RBP4 serum concentrations were lower in women with than in women without gestational diabetes. However, the western blot RBP4:retinol molar ratio was higher in women with gestational diabetes and negatively correlated with insulin levels post glucose load. In addition, western blot RBP4: retinol and EIA RBP4:retinol ratios correlated with fasting blood glucose in gestational diabetes. The potential importance of the RBP4:retinol ratio as a marker of metabolic disease is also illustrated in a study of normal-weight, overweight and obese children, in which the RBP4:retinol molar ratio correlated more strongly with components of the metabolic syndrome than serum RBP4 alone [28]. Since serum retinol levels are

Table 3 RBP4 measurements and other parameters as indicated in patients with and without gestational diabetes mellitus at median gestational week 30 and 8 weeks after delivery

\begin{tabular}{|c|c|c|c|c|}
\hline & \multicolumn{2}{|c|}{ Gestational diabetes $(n=20)$} & \multicolumn{2}{|c|}{ No gestational diabetes $(n=22)$} \\
\hline & During pregnancy & After delivery & During pregnancy & After delivery \\
\hline EIA RBP4 $(\mu \mathrm{g} / \mathrm{ml})$ & $6.7(3.3-14.4)^{\dagger}$ & $11.9(7.5-15.5)^{*}$ & $10.4(7.8-17.5)$ & $13(8.6-16.6)$ \\
\hline Western blot RBP4 $(\mu \mathrm{g} / \mathrm{ml})^{\mathrm{a}}$ & $25.5(21.7-30.7)$ & $27.0(23.4-35.9)^{*}$ & $27.3(24.0-32.2)$ & $27.9(24.0-34.1)$ \\
\hline Transthyretin $(\mu \mathrm{g} / \mathrm{ml})^{\mathrm{b}}$ & $161.9(119.8-172.6)$ & $164.5(132.8-236.6)$ & $176.8(132.8-200.1)$ & $141.7(127.1-207.8)$ \\
\hline RBP4:transthyretin ${ }^{\mathrm{c}}$ & $0.45(0.35-0.54)$ & $0.44(0.36-0.47)$ & $0.40(0.38-0.52)$ & $0.45(0.35-0.62)$ \\
\hline Retinol $(\mu \mathrm{mol} / \mathrm{l})$ & $1.00(0.66-1.22)$ & $2.47(1.89-3.14) *{ }^{\dagger}$ & $1.16(0.88-1.29)$ & $1.67(1.34-2.37) *$ \\
\hline RBP4:retinol ${ }^{\mathrm{d}}$ & $1.32(0.99-1.62)$ & $0.62(0.41-0.74) *$ & $1.41(0.88-1.60)$ & $0.76(0.55-0.89) *{ }^{\dagger}$ \\
\hline BMI $\left(\mathrm{kg} / \mathrm{m}^{2}\right)$ & $34(28-38)^{\dagger}$ & $32(25-35)^{*}{ }^{\dagger}$ & $27(24-29)$ & $24(22-28) *$ \\
\hline Systolic BP (mmHg) & $117(105-126)$ & $113(100-129)$ & $115(104-126)$ & $115(109-128)$ \\
\hline Diastolic BP (mmHg) & $74(67-79)$ & $79(70-85)$ & $73(67-77)$ & $76(70-83)$ \\
\hline Insulin $(\mathrm{pmol} / \mathrm{l})$ & $83(63-129)$ & $65(60-96)$ & $85(58-108)$ & $49(36-89)$ \\
\hline $1 \mathrm{~h}$ insulin $(\mathrm{pmol} / \mathrm{l})$ & $522(389-783)$ & $484(276-572)$ & $623(354-1276)$ & $276(188-522)^{*}$ \\
\hline $2 \mathrm{~h}$ insulin (pmol/1) & $808(597-864)$ & $292(222-438)^{*}$ & $569(322-876)$ & $218(126-343)^{*}$ \\
\hline HOMA-IR & $2.7(1.8-4.0)$ & $2.3(1.9-3.3)$ & $1.9(1.5-2.9)$ & $1.6(1.1-2.8)$ \\
\hline Glucose (mmol/l) & $5.0(4.5-5.6)^{\dagger}$ & $5.1(4.7-5.4)^{\dagger}$ & $4.1(3.8-4.4)$ & $4.7(4.6-5.0)^{*}$ \\
\hline $1 \mathrm{~h}$ glucose $(\mathrm{mmol} / \mathrm{l})$ & $12.0(11.2-13.4)^{\dagger}$ & $9.5(8.0-10.8)^{*},^{\dagger}$ & $7.7(6.4-9.5)$ & $5.5(5.0-7.8)^{*}$ \\
\hline $2 \mathrm{~h}$ glucose $(\mathrm{mmol} / \mathrm{l})$ & $9.6(8.9-10.9)^{\dagger}$ & $6.3(5.6-7.2)^{*}$ & $6.7(5.8-7.2)$ & $5.6(4.8-6.4)$ \\
\hline Triacylglycerol (mmol/l) & $2.1(1.9-3.3)$ & $1.0(0.8-1.9)^{*}$ & $2.0(1.6-2.2)$ & $0.8(0.7-1.14)^{*}$ \\
\hline $\operatorname{GFR}\left(\mathrm{ml} \mathrm{min} \min ^{-1} 1.73 \mathrm{~m}^{-2}\right)$ & $141(123-151)$ & $116(90-138)^{*}$ & $145(120-159)$ & $109(92-125)^{*}$ \\
\hline $\mathrm{HbA}_{1 \mathrm{c}}(\%)$ & $5.6(5.4-5.9)^{\dagger}$ & $5.6(5.2-6.0)^{\dagger}$ & $5.0(4.9-5.4)$ & $5.2(5.0-5.3)$ \\
\hline
\end{tabular}

Data are presented as medians (interquartile range)

ANOVA and Fisher least significant difference post hoc test were applied to test for significant differences within and between groups after logtransformation of skewed variables

${ }^{\mathrm{a}} n=19$

${ }^{\mathrm{b}} n=19$

${ }^{\mathrm{c}}$ Molar ratio, according to the amounts of RBP4 and transthyretin determined by western blotting, calculated using a molecular mass of $21 \mathrm{kDa}$ for RBP4 and $54 \mathrm{kDa}$ for transthyretin as a homotetramer $(n=19)$

${ }^{\mathrm{d}}$ Molar ratio, according to the amount of RBP4 determined by western blot and the amount of retinol determined by HPLC, calculated using a molecular mass of $21 \mathrm{kDa}$ for RBP4 and $0.286 \mathrm{kDa}$ for retinol $(n=19)$

${ }^{*} p<0.05$ vs during pregnancy; ${ }^{\dagger} p<0.05$ vs no gestational diabetes 
dramatically altered during pregnancy, the RBP4:retinol ratio appears to be a more appropriate way of assessing the relationship of RBP4 to metabolic status during pregnancy than RBP4 alone. In addition, the potential use of the RBP4:transthyretin molar ratio in pregnancy is illustrated by the fact that this ratio correlated with HOMA-IR, fasting insulin and BMI in healthy pregnant controls in our study, whereas no relationship could be found between RBP4 alone and insulin resistance. Possible explanations for this are discussed below.

In our study, retinol concentrations correlated significantly with western blot RBP4 levels in women with gestational diabetes. We found lower retinol levels in women with gestational diabetes than in healthy pregnant women. Since data indicate that RBP4 must be bound to retinol to be secreted from hepatocytes [18, 23], these lower retinol levels could lead to a reduction in serum RBP4 levels. Pregnancy-associated alterations in blood volume need to be considered when interpreting retinol and RBP4 measurements. The increase in plasma volume which occurs during pregnancy is positively associated with factors such as parity, higher birthweight and increased maternal pre-pregnancy BMI [29]. The gestational diabetes participants in our study had higher pre-pregnancy BMI, which would result in higher circulating blood volume and lower RBP4 and retinol levels than in pregnant controls. In addition, the reduced plasma volume post-partum could, at least partially, contribute to an increase in RBP4 and retinol concentrations after delivery. Previous data have shown that retinol and RBP4 levels are lower in pregnant women than in non-pregnant controls when expressed as a concentration, but not when expressed per gram of circulating protein [30]. This also could be due, at least in part, to the expanded blood volume during pregnancy. The importance of pregnancy-associated alterations in retinol homeostasis is also illustrated in lactating women, who exhibit alterations in retinol and RBP levels post-partum, with the highest RBP4 values occurring at 11 to 12 weeks post-partum and the lowest values at 15 to 17 weeks post-partum [31]. Therefore, in the present study, the retinol and RBP4 values obtained at 8 weeks postpartum may also have been influenced by the recent pregnancy. In addition, urinary retinol-binding protein excretion is increased during pregnancy, which could also lower plasma RBP4 concentrations [32]. In our study, GFR was comparable between women with and without gestational diabetes and can therefore be excluded as a cause for the results observed.

Most of the RBP4 in serum is bound to retinol. It is not known whether unbound apolipoprotein-RBP4 has different biological effects from bound RBP4. However, the proportion of RBP4 bound to retinol is decreased in pregnancy [30]. This altered RBP4:retinol ratio in pregnancy may reflect the possibility that a ligand other than retinol can bind to RBP4 and that this 'alternative ligand' may be associated with features of the metabolic syndrome. Other retinoids, such as retinaldehyde, have metabolic effects and have been shown to bind to RBP4 [33]. Most of serum RBP4 is also bound to transthyretin, which prevents glomerular filtration of RBP4. C-terminal truncated forms of RBP4 are thought to have decreased binding affinity for transthyretin, leading to excretion in the urine. These forms, which are abundant in urine, have also been detected in serum and levels can vary in different metabolic states [34]. It is not known whether these forms of RBP4 have the same biological activity as full-length RBP4. Thus, total RBP4 could be an inaccurate measure of biologically active RBP4.

Recently, a strong relationship between RBP4 and triacylglycerol was suggested [35]. Our findings revealed a weak association between western blot RBP4 levels and triacylglycerol only in pregnant controls. Triacylglycerol levels were comparable between pregnant women with and without gestational diabetes. Many [18-20], but not all [36-38] studies show a correlation between serum RBP4 levels and the magnitude of insulin resistance in nonpregnant adults and children. Our study did not find an association between total RBP4 and HOMA-IR, although the RBP4:transthyretin ratio correlated with HOMA-IR in non-diabetic pregnant women.

Pregnancy itself, even without gestational diabetes, is an insulin-resistant state as evidenced by the fact that it is associated with a $50 \%$ decrease in insulin-mediated glucose uptake [39]. To maintain euglycaemia in a normal pregnancy, insulin secretion increases by 200 to $250 \%$ [39], and women who do not have adequate islet secretory reserve become diabetic. Due, in part, to hormonal influences, the pathogenesis of insulin resistance during pregnancy is not entirely the same as for the insulin resistance that is characteristic of the metabolic syndrome. RBP4 may not be a 'common denominator' of all forms of insulin resistance. RBP4 can be affected by alterations of sex hormones, as evidenced by the fact that $17 \beta$-oestradiol can increase RBP4 mRNA expression and RBP4 secretion from human adipose tissue and women with polycystic ovary syndrome have elevated adipose tissue and serum RBP4 levels [40]. Furthermore, progesterone in the presence of oestradiol decreases RBP4 content in the female reproductive tract [41]. The higher progesterone levels found in women with gestational diabetes than in those without [4] may reduce RBP4 levels in women with gestational diabetes.

Our study does not agree with another one demonstrating that Chinese women with gestational diabetes had elevated serum RBP4 levels at 24 to 28 gestational weeks compared with healthy pregnant controls [42]. This report differs from our study due to the lower average BMI $\left(26.1 \mathrm{~kg} / \mathrm{m}^{2}\right.$ at 24 to 28 gestational weeks). As reported by Retnakaran et al. [43], BMI before pregnancy has a great effect on insulin resistance during pregnancy in Asian women than in Europid women. 
Recently, concerns have arisen about the accuracy of RBP4 measurements. A study showed that RBP4 measured by EIA is less precise than the gold standard for RBP4 determination by western blot and that many commercial assays had a reduced range for RBP4 values and undervalued them in comparison with western blot [27]. Therefore we applied both methods. The EIA measured lower RBP4 concentrations than the western blot. Furthermore, the magnitude of difference between gestational diabetes and no gestational diabetes was much greater with the EIA. These assay results may differ because of different antibodies used, because the western blot technique, but not the EIA involves denaturation of the samples as well as other technical considerations. Importantly, neither assay showed an elevation of RBP4 in gestational diabetes but the RBP4:retinol ratio was increased in gestational diabetes. Since RBP4 circulates in several forms in human serum, it is possible that only certain moieties are related to insulin resistance. As mentioned above, the RBP4:retinol ratio has been shown to correlate more strongly with metabolic syndrome than RBP4 alone [28].

In conclusion, serum RBP4 concentrations are not elevated in women with gestational diabetes compared with pregnant controls, although the RBP4:retinol ratio is elevated in the former, correlating with fasting blood glucose and negatively with insulin levels post glucose load. BMI, fasting insulin and HOMA-IR are significantly related to the RBP4:transthyretin molar ratio in healthy pregnant women, but not in women with gestational diabetes. However, total RBP4 and RBP4:transthyretin correlate with systolic BP in gestational diabetes. RBP4 alone is not associated with insulin resistance in pregnant women with or without gestational diabetes. Thus, the RBP4:retinol and RBP4:transthyretin ratios appear to better reflect parameters of glucose homeostasis and blood pressure during pregnancy than RBP4 levels alone.

Acknowledgements This work was supported by a scientific educational grant from Novo Nordisk Vienna and by NIH grants R01 DK43051 and T32 DK07516.

Duality of interest The authors declare that there is no duality of interest associated with this manuscript.

\section{References}

1. Metzger BE (1991) Summary and recommendations of the Third International Workshop Conference on Gestational Diabetes Mellitus. Diabetes 40(Suppl 2):197-201

2. Noussitou P, Monbaron D, Vial Y, Gaillard RC, Ruiz J (2005) Gestational diabetes mellitus and the risk of metabolic syndrome: a population-based study in Lausanne, Switzerland. Diabetes Metab 31:361-369

3. Bo S, Signorile A, Menato G et al (2005) C-reactive protein and tumor necrosis factor-alpha in gestational hyperglycemia. J Endocrinol Invest 28:779-786
4. Couch SC, Philipson EH, Bendel RB, Pujda LM, Milvae RA, Lammi-Keefe CJ (1998) Elevated lipoprotein lipids and gestational hormones in women with diet-treated gestational diabetes mellitus compared to healthy pregnant controls. J Diabetes its Complicat 12:1-9

5. Koukkou E, Watts GF, Lowy C (1996) Serum lipid, lipoprotein and apolipoprotein changes in gestational diabetes mellitus: a cross-sectional and prospective study. J Clin Pathol 49:634-637

6. Henry OA, Beischer NA (1991) Long-term implications of gestational diabetes for the mother. Bailliere's Clin Obstet Gynaecol 5:461-483

7. Endo S, Maeda K, Suto M et al (2006) Differences in insulin sensitivity in pregnant women with overweight and gestational diabetes mellitus. Gynecol Endocrinol 22:343-349

8. Buchanan TA, Xiang AH (2005) Gestational diabetes mellitus. J Clin Invest 115:485-491

9. Kautzky-Willer A, Pacini G, Tura A et al (2001) Increased plasma leptin in gestational diabetes. Diabetologia 44:164-172

10. Retnakaran R, Hanley AJ, Raif N, Connelly PW, Sermer M, Zinman B (2004) Reduced adiponectin concentration in women with gestational diabetes: a potential factor in progression to type 2 diabetes. Diabetes Care 27:799-800

11. Winkler G, Cseh K, Baranyi E et al (2002) Tumor necrosis factor system in insulin resistance in gestational diabetes. Diabetes Res Clin Pract 56:93-99

12. Retnakaran R, Hanley AJ, Raif N, Connelly PW, Sermer M, Zinman B (2003) C-reactive protein and gestational diabetes: the central role of maternal obesity. J Clin Endocrinol Metab $88: 3507-3512$

13. Dahlgren J (2006) Pregnancy and insulin resistance. Metab Syndr Relat Disord 4:149-152

14. Buchanan TA, Xiang A, Kjos SL, Watanabe R (2007) What is gestational diabetes? Diabetes Care 30:S105-S111

15. Shepherd PR, Kahn BB (1999) Glucose transporters and insulin action-implications for insulin resistance and diabetes mellitus. N Engl J Med 341:248-257

16. Tamori Y, Sakaue H, Kasuga M (2006) RBP4, an unexpected adipokine. Nat Med 12:30-31

17. Garvey WT, Maianu L, Zhu JH, Hancock JA, Golichowski AM (1993) Multiple defects in the adipocyte glucose transport system cause cellular insulin resistance in gestational diabetes. Heterogeneity in the number and a novel abnormality in subcellular localization of GLUT4 glucose transporters. Diabetes 42:1773-1785

18. Yang Q, Graham TE, Mody N et al (2005) Serum retinol binding protein 4 contributes to insulin resistance in obesity and type 2 diabetes. Nature 436:356-362

19. Graham TE, Yang Q, Bluher M et al (2006) Retinol-binding protein 4 and insulin resistance in lean, obese, and diabetic subjects. N Engl J Med 354:2552-2563

20. Cho YM, Youn BS, Lee $\mathrm{H}$ et al (2006) Plasma retinol-binding protein-4 concentrations are elevated in human subjects with impaired glucose tolerance and type 2 diabetes. Diabetes Care 29:2457-2461

21. Episkopou V, Maeda S, Nishiguchi S et al (1993) Disruption of the transthyretin gene results in mice with depressed levels of plasma retinol and thyroid hormone. Proc Natl Acad Sci U S A 90:2375-2379

22. van Bennekum AM, Wei S, Gamble MV et al (2001) Biochemical basis for depressed serum retinol levels in transthyretin-deficient mice. J Biol Chem 276:1107-1113

23. Bellovino D, Apreda M, Gragnoli S, Massimi M, Gaetani S (2003) Vitamin A transport: in vitro models for the study of RBP secretion. Mol Aspects Med 24:411-420

24. No authors listed (2002) Report of the Expert Committee on the Diagnosis and Classification of Diabetes Mellitus. Diabetes Care 25:5S-20S 
25. Matthews DR, Hosker JP, Rudenski AS, Naylor BA, Treacher DF, Turner RC (1985) Homeostasis model assessment: insulin resistance and beta-cell function from fasting plasma glucose and insulin concentrations in man. Diabetologia 28:412-419

26. Levey AS, Bosch JP, Lewis JB, Greene T, Rogers N, Roth D (1999) A more accurate method to estimate glomerular filtration rate from serum creatinine: a new prediction equation. Modification of Diet in Renal Disease Study Group. Ann Intern Med 130:461-470

27. Graham TE, Wason CJ, Bluher M, Kahn BB (2007) Shortcomings in methodology complicate measurements of serum retinol binding protein (RBP4) in insulin-resistant human subjects. Diabetologia 50:814-823

28. Aeberli I, Biebinger R, Lehmann R, L'Allemand D, Spinas GA, Zimmermann MB (2007) Serum retinol-binding protein 4 concentration and its ratio to serum retinol are associated with obesity and metabolic syndrome components in children. J Clin Endocrinol Metab 92:4359-4365

29. Faupel-Badger JM, Hsieh CC, Troisi R, Lagiou P, Potischman N (2007) Plasma volume expansion in pregnancy: implications for biomarkers in population studies. Cancer Epidemiol Biomarkers Prev 16:1720-1723

30. Sapin V, Alexandre MC, Chaib S et al (2000) Effect of vitamin A status at the end of term pregnancy on the saturation of retinol binding protein with retinol. Am J Clin Nutr 71:537-543

31. Cumming FJ, Briggs MH (1983) Changes in plasma vitamin A in lactating and non-lactating oral contraceptive users. Br J Obstet Gynaecol 90:73-77

32. Cheung CK, Lao T, Swaminathan R (1989) Urinary excretion of some proteins and enzymes during normal pregnancy. Clin Chem 35:1978-1980

33. Ziouzenkova O, Orasanu G, Sharlach M et al (2007) Retinaldehyde represses adipogenesis and diet-induced obesity. Nat Med 13:695-702
34. Kiernan UA, Tubbs KA, Nedelkov D, Niederkofler EE, Nelson RW (2002) Comparative phenotypic analyses of human plasma and urinary retinol binding protein using mass spectrometric immunoassay. Biochem Biophys Res Commun 297: 401-405

35. Lee DC, Lee JW, Im JA (2007) Association of serum retinol binding protein 4 and insulin resistance in apparently healthy adolescents. Metabolism 56:327-331

36. Erikstrup C, Mortensen OH, Pedersen BK (2006) Retinol-binding protein 4 and insulin resistance. N Engl J Med 355:1393-1394

37. Takashima N, Tomoike H, Iwai N (2006) Retinol-binding protein 4 and insulin resistance. N Engl J Med 355:1392

38. Janke J, Engeli S, Boschmann M et al (2006) Retinol-binding protein 4 in human obesity. Diabetes 55:2805-2810

39. Barbour LA, McCurdy CE, Hernandez TL, Kirwan JP, Catalano PM, Friedman JE (2007) Cellular mechanisms for insulin resistance in normal pregnancy and gestational diabetes. Diabetes Care 30(Suppl 2):S112-S119

40. Tan BK, Chen J, Lehnert H, Kennedy R, Randeva HS (2007) Raised serum, adipocyte and adipose tissue retinol-binding protein 4 in overweight women with polycystic ovary syndrome: effects of gonadal and adrenal steroids. J Clin Endocrinol Metab 92:2764-2792

41. Eberhardt DM, Jacobs WG, Godkin JD (1999) Steroid regulation of retinol-binding protein in the ovine oviduct. Biol Reprod 60:714-720

42. Chan TF, Chen HS, Chen YC et al (2007) Increased serum retinolbinding protein 4 concentrations in women with gestational diabetes mellitus. Reprod Sci 14:169-174

43. Retnakaran R, Hanley AJ, Connelly PW, Sermer M, Zinman B (2006) Ethnicity modifies the effect of obesity on insulin resistance in pregnancy: a comparison of Asian, South Asian, and Caucasian women. J Clin Endocrinol Metab 91:93-97 